\title{
RATIONAL USE OF MRI IN BACK PAIN
}

\author{
R. K. Jayshree ${ }^{1}$, O. Guneshwar Singh ${ }^{2}$
}

${ }^{1}$ Associate Professor, Department of Radiodiagnosis, Jawaharlal Nehru Institute of Medical Sciences, Porompat, Imphal, Manipur, India.

${ }^{2}$ Associate Professor, Department of Orthopaedics, Jawaharlal Nehru Institute of Medical Sciences, Porompat, Imphal, Manipur, India. ABSTRACT

\section{BACKGROUND}

Low back pain is one of the most common orthopaedic problems affecting up to 80 to 90 percent of adults at some point of time in their life. In spite of its high prevalence, spontaneous recovery usually occurs within a few weeks' time and even in those cases having features of nerve root irritation; in 90 percent of the cases, their symptoms improve within two months' time whatever is the modality of treatment, but improvement may take place even without treatment. MRI also demonstrates abnormality in people without back pain.

\section{METHODS}

This is a retrospective study conducted among 664 patients who were subjected to MRI at JNIMS, Imphal during January 2017 to December 2018.

\section{RESULTS}

Degenerative changes in the disc are the most common finding detected during the study. Other findings are spinal stenosis, fracture, infection, neoplasm and few other associated lesions.

\section{DISCUSSION}

MRI is usually not necessary in most cases of nonspecific back pain, as such cases usually recover within a period of 6-8 weeks. MRI, though, very sensitive for detection of degenerative changes, should not be suggested in the initial one month of onset of back pain. It should be indicated when there is severe or progressive neurological deficit.

\section{CONCLUSIONS}

Surgical intervention should not be done based on MRI findings alone \& without clinical correlation. MRI should be avoided in the absence of neurological deficit or other red flags.

\section{KEY WORDS}

Low Back Pain (LBP), MRI, Gradient Echo (GRE), Fast Spin Echo (FSE), Rational use of MRI, Herniated Disc, Sequestrated Disc, Sciatica, Spinal Claudication

HOW TO CITE THIS ARTICLE: Jayshree RK, Singh OG, Dixit S. Rational use of MRI in back pain. J. Evolution Med. Dent. Sci. 2019;8(30):2417-2422, DOI: 10.14260/jemds/2019/529

\section{BACKGROUND}

Low back pain is one of the most common problems encountered in daily orthopaedic outpatient department. It could be labelled as "everyone's problem" since it afflicts up to 80 to 90 percent of adults at some point of time in their life and up to 50 percent will have more than one attack ${ }^{1}$. In developed countries it is the second common cause of consulting a doctor and third common cause for surgical intervention. It has been reported that the lifetime prevalence of low back pain is around 60 to 70 percent. In spite of its high prevalence, usually in 60 percent of cases spontaneous recovery occurs within one week, 90 percent at six weeks and 95 percent in 3 months ${ }^{2}$. An accurate diagnosis can be made only in 10 to 20 percent of the patients.

'Financial or Other Competing Interest': None.

Submission 05-06-2019, Peer Review 09-07-2019,

Acceptance 15-07-2019, Published 29-07-2019.

Corresponding Author:

Dr. O. Guneshwar Singh,

Nepra Menjor Mamang Leikai,

Sagolband, Imphal-795001,

Manipur, India.

E-mail: singhguneshwar@gmail.com

DOI: $10.14260 /$ jemds $/ 2019 / 529$
In most cases, causes of low back pain are not obvious. Majority of the cases are due to mechanical causes involving injury to muscles, tendons, ligaments, deep fascia or bone. It is difficult to make an exact pathoanatomic diagnosis in cases like this. Usually, it is also not necessary to make a precise pathoanatomic diagnosis as pains originating from these different sources are treated in the same way even in acute phase and they do recover spontaneously within few weeks.

Back pain can also be caused by abnormalities of the organs in abdomen, pelvis or chest. Such referred pain can result from appendicitis, aneurysms, pelvic infections, bladder infections, kidney infections, kidney diseases, uterine fibroid, ovarian disorders and endometriosis. Normal pregnancy also causes pain in the back due to stretching of pelvic ligaments, irritating nerves and even causing strain of back muscles. Oestrogen and a ligament loosening hormone relaxin may be responsible for loosening of the ligaments and other structures of the back.

Nerve root syndromes are produced when a nerve is directly irritated due to a herniation or bulging of intervertebral disc. Sciatica is produced due to impingement of a nerve root. These types of pain are sharp in intensity, affect a particular area and are usually associated with numbness of the area of the leg supplied by the affected nerve. 
When disc degenerate or grow thinner, herniation or bulge develops. The central portion of the disc which is jelly like bulges out of the centre of the disc and presses on a nerve root. This degenerative process of disc usually begins by the $3^{\text {rd }}$ decade of life. In about one-third of adults over 20 years of age herniated disc are found and only about $3 \%$ of such herniation produces symptoms of nerve root irritation.

When intervertebral disc loses its moisture and volume due to ageing process decreasing its height, spondylosis occurs. In such cases even minor trauma can cause inflammation and nerve root irritation or impingement producing symptoms of sciatica without disc rupture.

When disc degeneration is associated with disease of joints of lower back it can lead to narrowing of spinal canal (spinal stenosis). These changes in the disc and joints can also be seen on X-ray. Patients suffering from the abovementioned symptoms may have pain radiating to both lower limbs while standing for a prolonged period or even when walking short distances.

When the spinal canal is directly compressed, a medical emergency called cauda equina syndrome results. Here, the disc material encroaches into spinal canal compressing nerve roots. Such a case would have pain, loss of sensation, loss of bladder or bowel function causing inability to control urination resulting in incontinence or inability to start urination.

Musculoskeletal causes of back pain include myofascial pain syndromes and fibromyalgia. In myofascial pain, there is pain and tenderness over localised areas or trigger points. There is also loss of range of motion in the involved muscle groups and the pain radiates in a characteristics distribution but restricted to a peripheral nerve. Stretching of the involved muscle group that may give pain relief. In fibromyalgia, there is widespread pain and tenderness throughout the body. There can also be generalised stiffness, fatigue and muscle aches.

Sometimes, sources of back pain may be from sacroiliac joint, hip joint and musculature. Other uncommon causes of low back pain may be infections of bones, pyogenic or tubercular osteomyelitis of spine. Non-infectious type of spinal inflammation i.e. spondylitis also causes pain and stiffness in lower back which is worse in the morning. Other sources of skeletal pain may be benign or malignant tumours, primary or metastatic tumours. Herpes zoster virus causing shingles can also cause inflammation of nerves from the spine. This lesion can cause pain in thoracic area (upper back pain) or in the lumbar area (low back pain).

Therefore, it is very essential to have a thorough medical evaluation before prescribing diagnostic tests. Even psychological and emotional factors particularly depression can play an important role in the causation of back pain. ${ }^{3}$

Back pain may be classified into 3 types based on the duration of symptoms. ${ }^{4}$

1. Acute back pain-duration of pain less than 6 weeks.

2. Sub-acute back pain-duration of pain more than 6 weeks but less than 12 weeks.

3. Chronic back pain-duration of pain is more than 12 weeks.

The main symptom of low back pain is pain in lumbosacral area. The pain may radiate to the front, side or back of the leg or the pain may remain confined to the lower back only. The pain may worsen with activity and occasionally the pain may worsen at night or with prolonged sitting. There can be numbness or weakness in the part of the leg supplied by the compressed nerve. There can be inability to plantarflex the foot. The patient would be unable to stand on one's toes and this occurs when the first sacral nerve is compressed or injured. The individual will be unable to raise the big toe when the fifth lumbar nerve root is compressed.

Eleven red flags have been identified by the Agency for Healthcare Research and quality 5 . The emphasis of these red flags is to identify fractures, infections or tumours of the spine. In presence of any of the following red flags associated with low back pain complete evaluation should be done.

\section{Red Flags}

1. Significant traumas of recent origin e.g. fall from height, motor vehicle accident or any other accident of similar nature.

2. History of mild trauma of recent origin in a patient over 50 years of age e.g. falls down by few steps or slipping and hitting on the buttocks.

3. Prolonged history of use of steroid e.g. use of steroid for rheumatic disorders, asthma or COPD, etc.

4. Individual with history of osteoporosis e.g. woman with history of hip fracture.

5. Individuals over 70 years of age. In such individuals there is increased chance of cancer, infections or other abdominal causes.

6. Previous history of cancer.

7. Recent history of any infection.

8. Individuals with temperature over $100^{\circ} \mathrm{F}$.

9. History of I.V. drug use. There is increased risk of an infectious cause.

10. Low back pain which is worse at night. Such cases are usually associated with infectious or malignant process but may also occur with ankylosing spondylitis.

11. Person having unexplained weight loss.

In presence of any of the above red flags in history the individual should visit the hospital for thorough examination and investigation. Immediate visit to hospital should be done in presence of any feature of acute nerve dysfunction. These patients may be unable to walk, unable to raise or lower foot at ankle. They may also be unable to raise big toe upward, walk on heels or unable to stand on the toes. All the above features indicate acute nerve injury or compression. Individuals having loss of bowel or bladder control including difficulty in initiating or stopping a stream of urine or having incontinence of urine requires urgent evaluation. Another indication for revaluation is that those patients who are not getting any benefit or relief from the medicines being taken.

Thorough history taking should include patient's age, constitutional symptoms, presence of nocturnal pain, bone pain, morning stiffness, occurrence of visceral pain, claudication symptoms, and neurological symptoms such as weakness, numbness, radiating pain and bowel and bladder dysfunction. We should also inquire about the characteristic and severity of the pain, history of trauma, previous medication or therapy and its effect and functional impact of the pain on patients work and other activities of daily living. An assessment should also be done on social and psychological factors like depression.

Initial evaluation of the patient includes thorough neurological examination of deep tendon reflexes, sensation, 
muscle strength. Peripheral pulses should be assessed, abdomen should be palpated to find out any organomegaly. Joint \& muscle flexibility of lower extremities should be assessed, entire spine be examined and also examine stance, gait, posture and straight leg raising test. Pain on forward flexion is one of the most common finding and usually indicates a mechanical cause. In case of pain induced by back extension spinal stenosis should be suspected. Evaluation of range of motion of spine has limited diagnostic value but it may be helpful in planning treatment and monitoring the patient ${ }^{6}$. An individual who is unable to walk heel to toe and squat and rise may have neurological involvement.

\section{Red Flags for Physical Examination}

1. Saddle shaped anaesthesia.

2. Loss of anal sphincter tone.

3. Major motor weakness in lower limbs.

4. Fever.

5. Tenderness over vertebrae.

6. Limitation in spinal range of motion.

7. Neurological findings present for more than one month.

8. Structural deformity in spine.

The prognosis of back pain is good in most cases and in 90 percent of cases including those having features of nerve root irritation their symptoms usually improves within 2 months whatever is the modality of treatment given and improvement also takes place even without any treatment.

MRI is considered to be the best imaging modality of choice for investigation of LBP due to high contrast, spatial resolution and lack of ionizing radiation. In spite of these MRI has many limitations and drawbacks. MRI \& CT scan also demonstrates abnormalities even in normal people without back pain.7,8 In another study of asymptomatic individuals MRI scans revealed herniated disc in about 25 percent cases in persons of age less than 60 years and 33 percent in persons of age more than 60 years. ${ }^{9}$ The presence of such positive findings does not correlate with patients symptoms. MRIs on normal people showed bulging disc in about $40 \%$ of the cases. MRI also failed to detect/ diagnose up to $20 \%$ cases of ruptured disc found during surgery. Therefore, every attempt should be made to correlate MRI findings with clinical features.

Intervertebral disc which allows relative motion of one vertebra over the other are cartilaginous structures. The central nucleus pulposus mainly consists of proteoglycan and is surrounded by annulus fibrous which is rich in collagen. The nucleus pulposus consists of about 80 percent water and with ageing there is desiccation or decrease in water content ${ }^{10}$. On imaging due to its water content nucleus pulposus appears hyperintense and annulus fibrosis hypointense on $\mathrm{T} 2$. With ageing as water content of the central position of the disc decreases, there is uniform hypointense appearance of the disc and this is the first imaging evidence of disc degeneration. ${ }^{11}$ The term "disc degeneration" includes desiccation, decreased disc height, disc bulging or herniation, fissures and calcification.

\begin{tabular}{|c|c|c|}
\hline Age & No. of Cases & Percentage \\
\hline Up to 20 years & 16 & 2.41 \\
\hline $21-30$ years & 110 & 16.57 \\
\hline $31-40$ years & 131 & 19.73 \\
\hline $41-50$ years & 156 & 23.49 \\
\hline 51-60 years & 132 & 19.88 \\
\hline Above 60 years & 119 & 17.92 \\
\hline \multicolumn{3}{|c|}{ Table I. Age Distribution } \\
\hline MRI Findings & No. of Cases & Percentage \\
\hline Normal & 30 & 4.52 \\
\hline Degenerative disc & 456 & 68.67 \\
\hline Canal stenosis & 130 & 19.58 \\
\hline \multirow{2}{*}{ Fractures } & 26 & 3.92 \\
\hline & 16 & 2.41 \\
\hline Neoplasm & 4 & 0.60 \\
\hline Others & 2 & 0.30 \\
\hline \multicolumn{3}{|c|}{ Table II. Distribution of MRI Findings } \\
\hline
\end{tabular}

\begin{tabular}{|c|c|c|}
\hline Associated Lesions & No. of Cases & Percentage \\
\hline Synovial cyst & 20 & 3.01 \\
\hline Haemangioma & 4 & 0.60 \\
\hline Schmorl's node & 6 & 0.90 \\
\hline Transitional vertebrae & 7 & 1.05 \\
\hline Spondylolisthesis & 14 & 2.10 \\
\hline \multicolumn{3}{|c|}{ Table III. Associated Lesions } \\
\hline DISC Level & No. of Cases & Percentage \\
\hline D12- L1 & 5 & 0.75 \\
\hline L1-L2 & 17 & 2.56 \\
\hline L2-L3 & 47 & 7.08 \\
\hline L3-L4 & 128 & 19.28 \\
\hline L4-L5 & 457 & 68.83 \\
\hline L5-S1 & 283 & 42.62 \\
\hline \multicolumn{3}{|c|}{ Table IV. Showing Affected Disc Level } \\
\hline
\end{tabular}

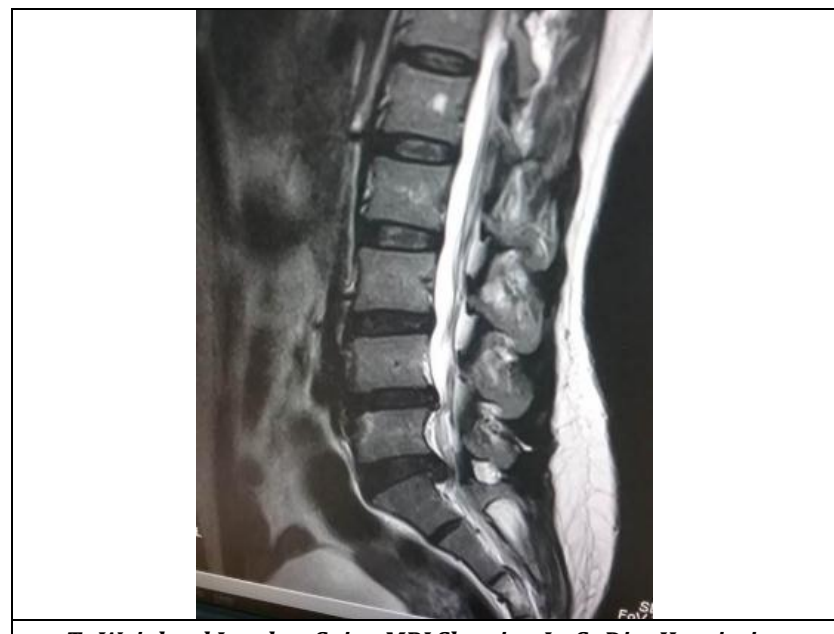

$T_{2}$ Weighted Lumbar Spine MRI Showing $L_{5}-S_{1}$ Disc Herniation

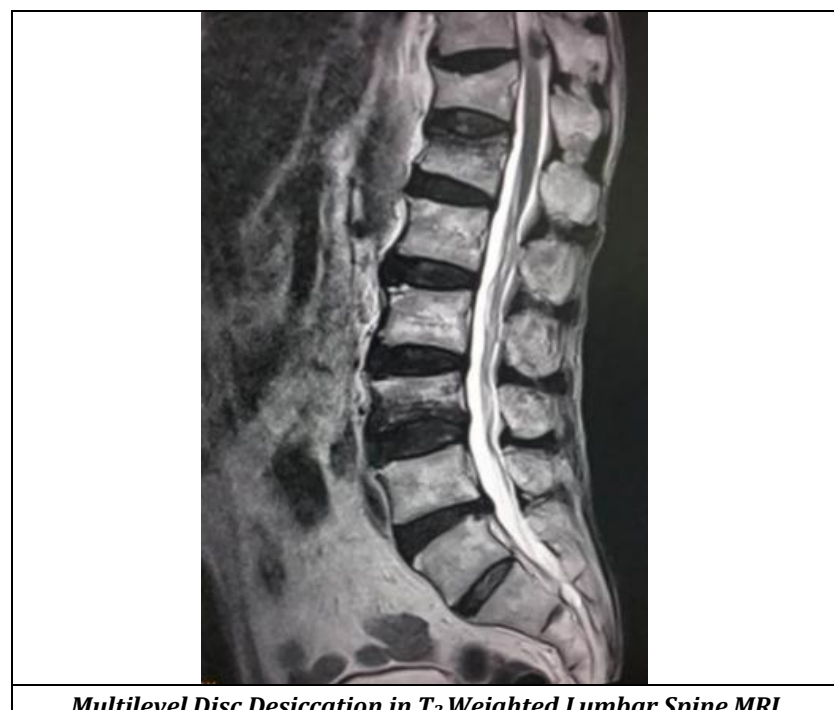

Multilevel Disc Desiccation in $T_{2}$ Weighted Lumbar Spine MRI 


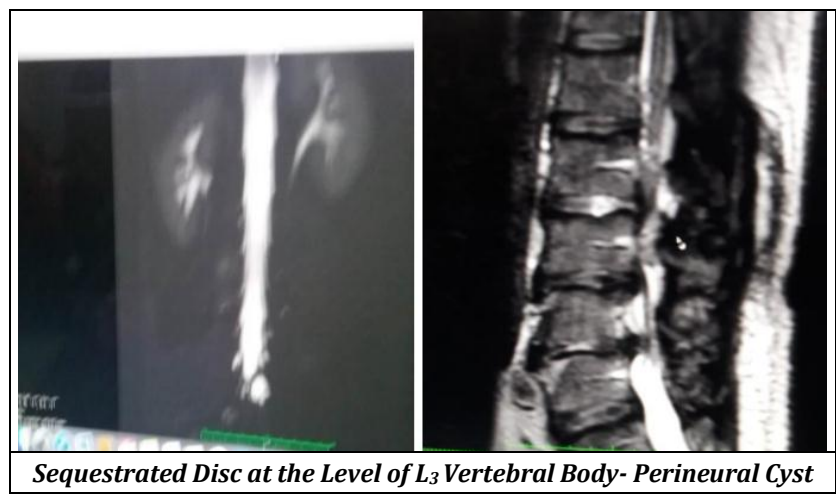

Pathologically disc degeneration is an inflammatory process and there are also associated changes in the adjacent endplates. Fissures indicate a focal loss in integrity of the annulus fibrosus. Disc bulge denotes enlargement or projection of disc beyond the margin of adjacent vertebral bodies circumferentially. Disc herniation which includes both protrusion or extrusion denote focal bulge of $3 \mathrm{~mm}$ or more beyond the margin of vertebral bodies. The term extrusion is used when the dome of the herniated position is wider than its attachment to the parent disc in any plane. If the extruded or sequestrated disc lie in a superior or inferior location compared to the parent disc it is said to have migrated. Sequestration is also used to denote a disc that have separated from the parent disc.

In 40 per cent cases of low back pain facet joint arthropathy may be responsible as they share the load of the spine with the disc. ${ }^{12}$ Facet joint arthropathy may also be responsible for degenerative spondylolisthesis and it may also be associated with thickening/calcification of ligamentum flavum complementing in narrowing the spinal canal. ${ }^{13}$ Degenerative lumbar stenosis follows due to change in the disc, facet and ligamentum flavum leading to encroachment upon the canal. The encroachment causes venous congestion and venous hypertension in the cauda equina leading to symptom of neurogenic intermittent claudication. ${ }^{14}$

This high prevalence of abnormal or irrelevant findings even in individual without LBP can result in emotional stress and even unnecessary surgical intervention. Some authors have reported higher rate of spinal surgery due to adoption of the advanced imaging technology which is not associated with better patient outcome. Though MRI is a method of choice for evaluation of disc pathology its sensitivity and specificity for disc herniations are only $60100 \%$ and 43-97 $\%$ respectively. High prevalence of disc degeneration and protrusion in asymptomatic adults is responsible for its lower specificity.

The prognosis of back pain is good in most cases and in $90 \%$ of cases including those having features of nerve root irritation, their symptoms usually improves within 2 months whatever is the modality of treatment given and improvement takes place even without any treatment.

Therefore, MRI should not be prescribed randomly unless there are conditions which need urgent surgical intervention like cauda equina syndrome, when there are red flags in physical examination or when there are features suggestive of spinal canal infection, tumour, fracture etc. which are responsible for less than $1 \%$ of all cases of back pain.

\section{METHODS}

The retrospective study was conducted in the department of radiodiagnosis and orthopaedics Jawaharlal Nehru Institute of Medical Sciences, Porompat, Imphal over a period of two years starting from January 2017 to Dec 2018. A total of 664 cases with history of back pain who have been subjected to MRI were taken up for the study. These are the patients who have been referred to the department for MRI lumbar spine in the investigation of back pain.

The MRI machine used in the department is 3.0 Tesla machine of Philips model Achiva. The spin echo (SE) is the standard pulse sequence used in the machine. The advantage of SE is that it has high signal to noise ratio and high contrast to noise ratio when appropriate parameters are used but they require relatively long acquisition time compare with gradient echo (GRE) and fast spin echo (FSE) technique. GRE technique is useful for evaluating post-operative spine cases.

In lumbar spine, SE and FSE imaging sequences are optimal in the evaluation of degenerative disorders as epidural and foraminal fat are abundant and gives out high intensity in $\mathrm{T}_{1} \mathrm{SE}$ compare to relatively low intensity of adjacent thecal sac, herniated disc or other tissue. In $\mathrm{T}_{2}$ weighted GRE \& FSE images the intensity of epidural fat is similar to or less than of CSF and also does not provide adequate contrast of other tissues in the spinal cord.

Disc pathology may be classified into disc bulge, disc herniation, disc protrusion, disc extrusion or sequestrated disc. Disc bulge is the enlargement or projection of the disc beyond the margin of adjacent vertebral bodies circumferentially whereas disc herniation is the focal bulge of $3 \mathrm{~mm}$ or more beyond the margin of vertebral bodies. Extruded disc is one in which the dome of the herniated portion is wider than its attachment to the parent disc in any plane. If the extruded disc lies in a superior or inferior location as compared to the plane of the parent disc it is known as migrated one. The fragment of a disc that has separated from the parent disc is known as sequestrated disc.

MRI findings in the study were categorised as based on the reports given by expert radiologist of the department as follows-

1. Normal.

2. Degenerated disc.

3. Spinal canal stenosis.

4. Fracture.

5. Infection.

6. Neoplasm.

7. Others.

\section{Statistical Analysis}

Descriptive Statistics like mean, proportion and standard deviation were used in the study.

\section{RESULTS}

The study consisted of 664 cases of back pain who have undergone MRI evaluation in the department. There were 395(59.5\%) male and $269(40.5 \%)$ female patients. The age wise distribution of cases is shown in table I. Maximum number of cases were in the age group of 41-50 years (23.5) followed by 51-61 years group (19.9\% ) and 31-41 years group $(19.7 \%)$. The mean age in the study was 44.56 years with a standard deviation of 14.12 years. 
Table II shows the distribution MRI findings. Degenerative changes in the disc are the most common findings being encountered in 456 (68.67\%) of the cases. There are 130 (19.57\%) cases of spinal stenosis. The other findings are fracture in $26(3.92 \%)$ cases followed infection is $16(2.41 \%)$ cases. Neoplasm was encountered in $4(0.60 \%)$ cases only. Associated lesions commonly found are synovial cyst in 20 (3.01\%) cases followed by Spondylolisthesis is 14 $(2.10 \%)$ cases and transitional vertebrae in $7(1.05 \%)$ cases. Haemangioma and Schmorl's mode was found in less than $1.0 \%$ of the total cases under study. The distribution of associated lesion is shown in Table III.

The most commonly involved disc level is $\mathrm{L}_{4-5}$ disc space, which is affected in 457 (68.83\%) cases. This is followed by $\mathrm{L}_{5}-\mathrm{S}_{1}$ disc space which is found to be affected in 283 $(42.62 \%)$ of the cases. $\mathrm{D}_{12}-\mathrm{L}_{1}$ was the least affected disc involved in only $5(0.75 \%)$ cases. Table IV shows the involvement of different disc levels.

\section{DISCUSSION}

MRI is usually not necessary in most cases of non-specific low back pain because such cases of back pain usually resolves spontaneously within a period of 6-8 weeks. The use of MRI in cases like these has been shown not to alter management of patients suffering from nonspecific low back pain. ${ }^{15,16}$ MRI though very sensitive for detection of degenerative changes should not be prescribed in the initial one month of onset of acute low back pain. MRI of lumbar spine is usually indicated when there is severe or progressive neurological deficit. Common indications for referring the patient for MRI of lumbar spine in patients presenting with low back pain should be-

1. Sciatic pain radiating below the knee.

2. Positive straight leg raising or positive femoral stretch test for more than 6 weeks duration.

3. Sciatica associated with motor deficit.

4. Spinal claudication with pain, weakness or numbness in both legs which have been presenting for more than 6 weeks when walking but eased on forward bending or sitting.

MRI is the method of choice when spinal infection is suspected because of its high sensitivity and specificity. ${ }^{17}$ Signal changes occur in the early part of the disease process. Short time inversion recovery sequence (STIR) is able to detect inflammatory oedema as the site of initial infective focus. ${ }^{18}$ Early diagnosis of spondylitis helps in avoiding severe spinal or neurological complications. MRI is more accurate than plain X-ray or bone scan for detection of osteomyelitis. It has been reported to be able to detect infection within 3-5 days of its onset and also have reported sensitivity of $96 \%$ and specificity of $92 \%$ for diagnosis of spinal infections. It is also one of the most accurate method of detecting malignancy and metastatic deposit in vertebrae. It is also very helpful in distinguishing benign from malignant compression fractures. For detection of spinal stenosis, it has a reported sensitivity of 87-96\% and specificity of $68-75 \%$.

Out of the 664 cases who are being subjected to MRI examination only 26 (3.91\%) patients underwent surgical intervention. 20 patients were operated for disc prolapsed, 2 patients for canal stenosis, 2 cases for spondylolisthesis and 1 each for cauda equina syndrome and compression fracture.
As most patients with non-specific back pain usually recover on conservative treatment, the primary indication for lumbar spine MRI in patients having back pain of recent onset should be to rule out serious or life-threatening conditions. The high percentage of disc changes even in individuals without back pain have raised the question whether MRI has exaggerated the prevalence of disc related cause of low back pain.9,19,20

\section{CONCLUSIONS}

Most cases of back pain are benign in nature and neurological impairment does not occur. Careful history taking and physical examination are very important in identifying systemic disease. In the absence of evidence of systemic disease, MRI should be delayed until 6 weeks of conservative treatment. In patients having sciatica, or those having symptoms of spinal stenosis, MRI should be prescribed only when symptoms don't improve with 6 weeks of conservative treatment. It not only gives accurate result for diagnosis of disc herniation and spinal stenosis but is also very sensitive for detecting infections and malignancies. MRI should be immediately done when there are features suggestive of cauda equina syndrome. There may also be many incidental findings on MRI and surgical interventions should not be undertaken based on such MRI findings alone without clinical correlation. It must always be used as an adjunct to clinical diagnosis not just treat the MRI findings. Hence, MRI should be avoided in the absence of neurological deficit or other red flags. So, we should treat patients not images.

\section{REFERENCES}

[1] Frymoyer JW. Back pain and sciatica. N Engl J Med 1988;318(5):291-300.

[2] Deyo RA, Weinstein JN. Low back pain. N Engl J Med 2001;344(5):363-70.

[3] Pincus T, Burton AK, Vogel S, et al. A systematic review of psychological factors as predictors of chronicity/disability in prospective cohorts of low back pain. Spine (Phila Pa 1976) 2002;27(5):E109-20.

[4] Carey TS, Garrett J, Jackman A, et al. The outcomes and costs of care for acute low back pain among patients seen by primary care practitioners, chiropractors and orthopaedic surgeons. The North Carolina Back pain Project. N Engl J Med 1995;333(14):913-7.

[5] Shiel WC Jr. Back Pain in 2nd $\& 3^{\text {rd }}$ Decades of life. In: John K, edr. Primer on the rheumatic diseases. $13^{\text {th }}$ edn. New York: Springer 2008.

[6] Lowery WD Jr, Hon TJ, Boden SD, et al. Impairment evaluation based on spinal range of motion subjects. J Spinal Disord 1992;5(4):398-402.

[7] Boden SD, Davis DO, Dina TS, et al. Abnormal magnetic-resonance scans of the lumbar spine in asymptomatic subjects. A prospective investigation. J Bone Joint Surg Am 1990;72(3):403-8.

[8] Wiesel SW, Tsourmas N, Feffer HL, et al. A study of computer-assisted tomography, I. The incidence of positive CAT scans in an asymptomatic group of patients. Spine 1984;9(6):549-51.

[9] Jensen MC, Brant-Zawadzki MN, Obuchowski N, et al. Magnetic resonance imaging of the lumbar spine in people without back pain. $\mathrm{N}$ Engl J Med 1994;331(2):69-73. 
[10] Roberts S, Evans H, Trivedi J, et al. Histology and pathology of the human intervertebral disc. J Bone Joint Surg Am 2006;88(Suppl 2):10-14.

[11] Videman T, Nummi P, Battie $M C$, et al. Digital assessment of MRI for lumbar disc desiccation. A comparison of digital versus subjective assessments and digital intensity profiles versus discogram and macroanatomic findings. Spine (Phila Pa 1976) 1994;19(2):192-8.

[12] Beresford ZM, Kendall RW, Willick SE. Lumbar facet syndromes. Curr Sports Med Rep 2010;9(1):50-6.

[13] Clarencon F, Law-Ye B, Bienvenot $P$, et al. The degenerative spine. Magnetic resonance imaging clinics of North America. Clin Rev Articles 2016;24(3):495-513.

[14] Amundsen T, Weber H, Nordal HJ, et al. Lumbar spinal stenosis: conservative or surgical management? a prospective 10-year study. Spine (Phila Pa 1976) 2000;25(11):1424-35.
[15] Gilbert FJ, Grant Am, Gillan MG, et al. Low back pain: influence of early MR imaging or CT on treatment an outcome-multicenter randomized trial. Radiology 2004;231(2):343-51.

[16] Jarvik JG, Hollingworth W, Martin B, et al. Rapid magnetic resonance imaging vs radiographs for patients with low back pain: a randomized controlled trial. JAMA 2003;289(21):2810-8.

[17] Cormican L, Hammal R, Messenger J, et al. Current difficulties in the diagnosis and management of spinal tuberculosis. Postgrad Med J 2006;82(963):46-51.

[18] Jevtic V. Vertebral infection. Eur Radiol 2004;14(Suppl 3):E43-E52.

[19] Paajanen H, Erkintalo M, Kuusela T, et al. Magnetic resonance study of disc degeneration in young lowback pain patients. Spine 1989;14(9):982-5.

[20] Powell MC, Wilson M, Szypryt P, et al. Prevalence of lumbar disc degeneration observed by magnetic resonance in symptomless women. Lancet 1986;2(8520):1366-7. 